\title{
Applications of Description Logics: State of the Art and Research Challenges
}

\author{
Ian Horrocks \\ School of Computer Science, University of Manchester \\ Oxford Road, Manchester M13 9PL, UK \\ horrocks@cs.man.ac.uk
}

\begin{abstract}
Description Logics (DLs) are a family of class based knowledge representation formalisms characterised by the use of various constructors to build complex classes from simpler ones, and by an emphasis on the provision of sound, complete and (empirically) tractable reasoning services. They have a range of applications, but are mostly widely known as the basis for ontology languages such as OWL. The increasing use of DL based ontologies in areas such as e-Science and the Semantic Web is, however, already stretching the capabilities of existing DL systems, and brings with it a range of challenges for future research.
\end{abstract}

\section{Introduction}

Description Logics (DLs) are a family of class (concept) based knowledge representation formalisms. They are characterised by the use of various constructors to build complex concepts from simpler ones, an emphasis on the decidability of key reasoning tasks, and by the provision of sound, complete and (empirically) tractable reasoning services.

Description logics have been used in a range of applications, e.g., configuration [1], and reasoning with database schemas and queries [2-4]. They are, however, best known as the basis for ontology languages such as OIL, DAML+OIL and OWL [5]. As well as DLs providing the formal underpinnings for these languages (i.e., a declarative semantics), DL systems are widely used to provide computational services for a rapidly expanding range of ontology tools and applications [6-11].

Ontologies, and ontology based vocabularies, are used to provide a common vocabulary together with computer-accessible descriptions of the meaning of relevant terms and relationships between these terms. Ontologies play a major role in the Semantic Web $[12,13]$, and are widely used in, e.g., knowledge management systems, e-Science, and bio-informatics and medical terminologies [14-17]. They are also of increasing importance in the Grid, where they may be used, e.g., to support the discovery, execution and monitoring of Grid services [18-20].

The success of the current generation of DLs and DL reasoning brings with it, however, requirements for reasoning support which may be beyond the capability of existing systems. These requirements include greater expressive power, 
improved scalability and extended reasoning services. Satisfying these requirements presents a major research challenge, not only to the DL community, but to the logic based Knowledge Representation community as a whole.

\section{Ontology Languages and Description Logics}

The OWL recommendation actually consists of three languages of increasing expressive power: OWL Lite, OWL DL and OWL Full. Like OWL's predecessor DAML+OIL, OWL Lite and OWL DL are basically very expressive description logics with an RDF syntax. OWL Full provides a more complete integration with RDF, but its formal properties are less well understood, and key inference problems would certainly be much harder to compute. ${ }^{1}$ For these reasons, OWL Full will not be considered in this paper.

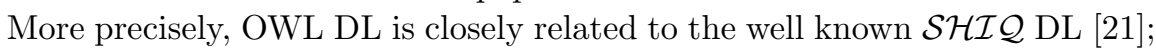
it restricts the form of $\mathcal{S H \mathcal { L }}$ number restrictions to be unqualified (see [22]), and extends $\mathcal{S H I} \mathcal{L}$ with nominals [23] (i.e., concepts having exactly one instance) and datatypes (often called concrete domains in DLs [24]). Following the usual DL naming conventions, the resulting logic is called $\mathcal{S H O \mathcal { I N }}(\mathbf{D})$ (where $\mathcal{O}$ stands for nominals, $\mathcal{N}$ stands for unqualified number restrictions and (D) stands for datatypes). OWL Lite is equivalent to the slightly simpler $\mathcal{S H I F}(\mathbf{D})$ DL. These equivalences allow OWL to exploit the considerable existing body of description logic research, e.g.:

- to define the semantics of the language and to understand its formal properties, in particular the decidability and complexity of key inference problems $[25]$;

- as a source of sound and complete algorithms and optimised implementation techniques for deciding key inference problems [21,26];

- to use implemented DL systems in order to provide (partial) reasoning support [27-29].

\section{$2.1 \mathcal{S H O I N}$ Syntax and Semantics}

The syntax and semantics of $\mathcal{S H O I N}$ are briefly introduced here (we will ignore datatypes, as adding a datatype component would complicate the presentation and has little affect on reasoning [30]).

Definition 1. Let $\mathbf{R}$ be a set of role names with both transitive and normal role names $\mathbf{R}_{+} \cup \mathbf{R}_{\mathrm{P}}=\mathbf{R}$, where $\mathbf{R}_{\mathrm{P}} \cap \mathbf{R}_{+}=\emptyset$. The set of $\mathcal{S H O I N}$-roles (or roles for short) is $\mathbf{R} \cup\left\{R^{-} \mid R \in \mathbf{R}\right\}$. A role inclusion axiom is of the form $R \sqsubseteq S$, for two roles $R$ and $S$. A role hierarchy is a finite set of role inclusion axioms.

\footnotetext{
${ }^{1}$ Inference in OWL Full is clearly undecidable as OWL Full does not include restrictions on the use of transitive properties which are required in order to maintain decidability [21].
} 
An interpretation $\mathcal{I}=\left(\Delta^{\mathcal{I}},{ }^{\mathcal{I}}\right)$ consists of a non-empty set $\Delta^{\mathcal{I}}$, called the domain of $\mathcal{I}$, and a function. ${ }^{\mathcal{I}}$ which maps every role to a subset of $\Delta^{\mathcal{I}} \times \Delta^{\mathcal{I}}$ such that, for $P \in \mathbf{R}$ and $R \in \mathbf{R}_{+}$,

$$
\begin{gathered}
\langle x, y\rangle \in P^{\mathcal{I}} \text { iff }\langle y, x\rangle \in P^{-\mathcal{I}}, \\
\text { and if }\langle x, y\rangle \in R^{\mathcal{I}} \text { and }\langle y, z\rangle \in R^{\mathcal{I}} \text {, then }\langle x, z\rangle \in R^{\mathcal{I}} .
\end{gathered}
$$

An interpretation $\mathcal{I}$ satisfies a role hierarchy $\mathcal{R}$ iff $R^{\mathcal{I}} \subseteq S^{\mathcal{I}}$ for each $R \sqsubseteq S \in \mathcal{R}$; such an interpretation is called a model of $\mathcal{R}$.

Definition 2. Let $N_{C}$ be a set of concept names with a subset $N_{I} \subseteq N_{C}$ of nominals. The set of $\mathcal{S H O I N}$-concepts (or concepts for short) is the smallest set such that

1. every concept name $C \in N_{C}$ is a concept,

2. if $C$ and $D$ are concepts and $R$ is a role, then $(C \sqcap D),(C \sqcup D),(\neg C)$, $(\forall R . C)$, and $(\exists R . C)$ are also concepts (the last two are called universal and existential restrictions, resp.), and

3. if $R$ is a simple role $e^{2}$ and $n \in \mathbb{N}$, then $\leqslant n R$ and $\geqslant n R$ are also concepts (called atmost and atleast number restrictions).

The interpretation function.$^{\mathcal{I}}$ of an interpretation $\mathcal{I}=\left(\Delta^{\mathcal{I}}, \cdot^{\mathcal{I}}\right)$ maps, additionally, every concept to a subset of $\Delta^{\mathcal{I}}$ such that

$$
\begin{aligned}
& (C \sqcap D)^{\mathcal{I}}=C^{\mathcal{I}} \cap D^{\mathcal{I}}, \quad(C \sqcup D)^{\mathcal{I}}=C^{\mathcal{I}} \cup D^{\mathcal{I}}, \quad \neg C^{\mathcal{I}}=\Delta^{\mathcal{I}} \backslash C^{\mathcal{I}}, \\
& (\exists R . C)^{\mathcal{I}}=\left\{x \in \Delta^{\mathcal{I}} \mid \text { There is a } y \in \Delta^{\mathcal{I}} \text { with }\langle x, y\rangle \in R^{\mathcal{I}} \text { and } y \in C^{\mathcal{I}}\right\}, \\
& (\forall R . C)^{\mathcal{I}}=\left\{x \in \Delta^{\mathcal{I}} \mid \text { For all } y \in \Delta^{\mathcal{I}}, \text { if }\langle x, y\rangle \in R^{\mathcal{I}}, \text { then } y \in C^{\mathcal{I}}\right\}, \\
& \leqslant n R^{\mathcal{I}}=\left\{x \in \Delta^{\mathcal{I}} \mid \sharp\left\{y \mid\langle x, y\rangle \in R^{\mathcal{I}}\right\} \leqslant n\right\}, \\
& \geqslant n R^{\mathcal{I}}=\left\{x \in \Delta^{\mathcal{I}} \mid \sharp\left\{y \mid\langle x, y\rangle \in R^{\mathcal{I}}\right\} \geqslant n\right\},
\end{aligned}
$$

where, for a set $M$, we denote the cardinality of $M$ by $\sharp M$.

For $C$ and $D$ (possibly complex) concepts, $C \sqsubseteq D$ is called a general concept inclusion (GCI), and a finite set of GCIs is called a TBox.

An interpretation $\mathcal{I}$ satisfies a GCI $C \sqsubseteq D$ if $C^{\mathcal{I}} \subseteq D^{\mathcal{I}}$, and $\mathcal{I}$ satisfies a TBox $\mathcal{T}$ if $\mathcal{I}$ satisfies each $G C I$ in $\mathcal{T}$; such an interpretation is called a model of $\mathcal{T}$.

$A$ concept $C$ is called satisfiable with respect to a role hierarchy $\mathcal{R}$ and a TBox $\mathcal{T}$ if there is a model $\mathcal{I}$ of $\mathcal{R}$ and $\mathcal{T}$ with $C^{\mathcal{I}} \neq \emptyset$. Such an interpretation is called a model of $C$ w.r.t. $\mathcal{R}$ and $\mathcal{T}$. A concept $D$ subsumes a concept $C$ w.r.t. $\mathcal{R}$ and $\mathcal{T}$ (written $C \sqsubseteq \mathcal{R}, \mathcal{T} D$ ) if $C^{\mathcal{I}} \subseteq D^{\mathcal{I}}$ holds in every model $\mathcal{I}$ of $\mathcal{R}$ and $\mathcal{T}$. Two concepts $C, D$ are equivalent w.r.t. $\mathcal{R}$ and $\mathcal{T}$ (written $C \equiv_{\mathcal{R}, \mathcal{T}} D$ ) iff they are mutually subsuming w.r.t. $\mathcal{R}$ and $\mathcal{T}$. (When $\mathcal{R}$ and $\mathcal{T}$ are obvious from the context, we will often write $C \sqsubseteq D$ and $C \equiv D$.) For an interpretation $\mathcal{I}$, an individual $x \in \Delta^{\mathcal{I}}$ is called an instance of a concept $C$ iff $x \in C^{\mathcal{I}}$.

${ }^{2}$ A role is simple if it is neither transitive nor has any transitive subroles. Restricting number restrictions to simple roles is required in order to yield a decidable logic [21]. 
Note that, as usual, subsumption and satisfiability can be reduced to each other, and reasoning w.r.t. general TBoxes and role hierarchies can be reduced to reasoning w.r.t. role hierarchies only $[21,26]$.

\section{$2.2 \quad$ Practical Reasoning Services}

Most modern DL systems use tableaux algorithms to test concept satisfiability. These algorithms work by trying to construct (a tree representation of) a model of the concept, starting from an individual instance. Tableaux expansion rules decompose concept expressions, add new individuals (e.g., as required by $\exists R . C$ terms), ${ }^{3}$ and merge existing individuals (e.g., as required by $\leqslant n R$. $C$ terms). Nondeterminism (e.g., resulting from the expansion of disjunctions) is dealt with by searching the various possible models. For an unsatisfiable concept, all possible expansions will lead to the discovery of an obvious contradiction known as a clash (e.g., an individual that must be an instance of both $A$ and $\neg A$ for some concept $A$ ); for a satisfiable concept, a complete and clash-free model will be constructed [31].

Tableaux algorithms have many advantages. It is relatively easy to design provably sound, complete and terminating algorithms, and the basic technique can be extended to deal with a wide range of class and role constructors. Moreover, although many algorithms have a higher worst case complexity than that of the underlying problem, they are usually quite efficient at solving the relatively easy problems that are typical of realistic applications.

Even in realistic applications, however, problems can occur that are much too hard to be solved by naive implementations of theoretical algorithms. Modern DL systems, therefore, include a wide range of optimisation techniques, the use of which has been shown to improve typical case performance by several orders of magnitude [32-34, 29, 35, 36]. Key techniques include lazy unfolding, absorption and dependency directed backtracking.

Lazy Unfolding In an ontology, or DL Tbox, large and complex concepts are seldom described monolithically, but are built up from a hierarchy of named concepts whose descriptions are less complex. The tableaux algorithm can take advantage of this structure by trying to find contradictions between concept names before adding expressions derived from Tbox axioms. This strategy is known as lazy unfolding [32,34].

The benefits of lazy unfolding can be maximised by lexically normalising and naming all concept expressions and, recursively, their sub-expressions. An expression $C$ is normalised by rewriting it in a standard form (e.g., disjunctions are rewritten as negated conjunctions); it is named by substituting it with a new concept name $A$, and adding an axiom $A \equiv C$ to the Tbox. The normalisation step allows lexically equivalent expressions to be recognised and identically named, and can even detect syntactically "obvious" satisfiability and unsatisfiability.

\footnotetext{
${ }^{3}$ Cycle detection techniques known as blocking may be required in order to guarantee termination.
} 


\begin{abstract}
Absorption Not all axioms are amenable to lazy unfolding. In particular, so called general concept inclusions (GCIs), axioms of the form $C \sqsubseteq D$ where $\mathrm{C}$ is non-atomic, must be dealt with by explicitly making every individual in the model an instance of $D \sqcup \neg C$. Large numbers of GCIs result in a very high degree of non-determinism and catastrophic performance degradation [34].

Absorption is another rewriting technique that tries to reduce the number of GCIs in the Tbox by absorbing them into axioms of the form $A \sqsubseteq C$, where $A$ is a concept name. The basic idea is that an axiom of the form $A \sqcap D \sqsubseteq D^{\prime}$ can be rewritten as $A \sqsubseteq D^{\prime} \sqcup \neg D$ and absorbed into an existing $A \sqsubseteq C$ axiom to give $A \sqsubseteq C \sqcap\left(D^{\prime} \sqcup \neg D\right)$ [37]. Although the disjunction is still present, lazy unfolding ensures that it is only applied to individuals that are already known to be instances of $A$.
\end{abstract}

Dependency Directed Backtracking Inherent unsatisfiability concealed in sub-expressions can lead to large amounts of unproductive backtracking search known as thrashing. For example, expanding the expression $\left(C_{1} \sqcup D_{1}\right) \sqcap \ldots \sqcap\left(C_{n} \sqcup\right.$ $\left.D_{n}\right) \sqcap \exists R .(A \sqcap B) \sqcap \forall R . \neg A$ could lead to the fruitless exploration of $2^{n}$ possible expansions of $\left(C_{1} \sqcup D_{1}\right) \sqcap \ldots \sqcap\left(C_{n} \sqcup D_{n}\right)$ before the inherent unsatisfiability of $\exists R$. $(A \sqcap B) \sqcap \forall R . \neg A$ is discovered. This problem is addressed by adapting a form of dependency directed backtracking called backjumping, which has been used in solving constraint satisfiability problems [38].

Backjumping works by labelling concepts with a dependency set indicating the non-deterministic expansion choices on which they depend. When a clash is discovered, the dependency sets of the clashing concepts can be used to identify the most recent non-deterministic expansion where an alternative choice might alleviate the cause of the clash. The algorithm can then jump back over intervening non-deterministic expansions without exploring any alternative choices. Similar techniques have been used in first order theorem provers, e.g., the "proof condensation" technique employed in the HARP theorem prover [39].

\title{
3 Research Challenges for Ontology Reasoning
}

Ontology based applications will critically depend on the provision of efficient reasoning support: on the one hand, such support is required by applications in order to exploit the semantics captured in ontologies; on the other hand, such support is required by ontology engineers to design and maintain sound, well-balanced ontologies. Experience with a wide range of applications and the development of user-oriented environments has highlighted a number of key requirements that will need to be met by the next generation of DL reasoners if they are to provide the basis for this support:

Greater expressive power For example, in ontologies describing complex physically structured domains such as biology [40] and medicine [41], it is often important to describe aggregation relationships between structures and their component parts, and to assert that certain properties of the component 
parts transfer to the structure as a whole (a femur with a fractured shaft is a fractured femur) [42]. The importance of this kind of knowledge can be gauged from the fact that various "work-arounds" have been described for use with ontology languages that cannot express it directly [43].

Similarly, in grid and web services applications, it may be necessary to describe composite processes in terms of their component parts, and to express relationships between the properties of the various components and those of the composite process. For example, in a sequential composition of processes it may be useful to express a relationship between the inputs and outputs of the composite and those of the first and last component respectively, as well as relationships between the outputs and inputs of successive components [13].

Improved scalability Practical ontologies may be very large - tens or even hundreds of thousands of classes. Dealing with large-scale ontologies already presents a challenge to the current generation of DL reasoners, in spite of the fact that many existing large-scale ontologies are relatively simple. In the 40,000 concept Gene Ontology (GO), for example, much of the semantics is currently encoded in class names such as "heparin-metabolism"; enriching GO with more complex definitions, e.g., by explicitly modelling the fact that heparin-metabolism is a kind of "metabolism" that "acts-on" the carbohydrate "heparin", would make the semantics more accessible, and would greatly increase the value of GO by enabling new kinds of query such as "what biological processes act on glycosaminoglycan" (heparin is a kind of glycosaminoglycan) [40]. However, adding more complex class definitions can cause the performance of existing reasoners to degrade to the point where it is no longer acceptable to users. Similar problems have been encountered with large medical terminology ontologies, such as the GALEN ontology [41]. As well as using a conceptual model of the domain, many applications will also need to deal with very large volumes of instance data-the GO, for example, is used to annotate millions of individuals, and practitioners want to answer queries that refer both to the ontology and to the relationships between these individuals, e.g., "what DNA binding products interact with insulin receptors". Answering this query requires a reasoner not only to identify individuals that are (perhaps only implicitly) instances of DNA binding products and of insulin receptors, but also to identify which pairs of individuals are (perhaps only implicitly) instances of the interactsWith role. For existing ontology languages it is possible to use DL reasoning to answer such queries, but dealing with the large volume of GO annotated gene product data is far beyond the capabilities of existing DL systems [44]. A requirement to store and query over large numbers of individuals is common in many application areas, e.g., in the Semantic Web, where ontologies are to be used in the annotation of web resources, and where users may want to answer queries such as "which bioinformatics researchers work in a university department where there is also a DL researcher?".

Extended reasoning services The ability to explain unexpected inferences is also crucial in ontology development and would be useful in query an- 
swering: when the DL reasoner returns an unexpected answer, such as an unintended sub-class relationship, users often find it difficult to understand (and if necessary fix) the causes of the unexpected inference; expert users may even doubt the validity of such inferences, and lose confidence in the inference system. Moreover, existing DL reasoners provide only a limited range of reasoning services, such as class subsumption and instance retrieval. In practice, users often want to ask questions for which it may only be possible to provide an approximate answer. For example, the user may want to be told "everything" that can be inferred about a class or individual, what it is "reasonable" to assert about a class or individual, or what the difference is between two classes or two individuals.

The following sections highlight interesting work in progress that addresses some of the above problems; they do not constitute an exhaustive survey.

\subsection{Expressive Power}

Some of the above mentioned requirements may be met by tableau algorithms that have recently been developed for DLs that are more expressive than those currently implemented in state-of-the-art reasoners. These algorithms are able to deal with, e.g., nominals (singleton classes) [26], complex role inclusion axioms [45], the use of datavalues as keys [46], the representation of temporal constraints [47], and the integration of reasoning over datatypes and built-in predicates [48]. The increased expressive power of these DLs would satisfy (at least partially) key application requirements, e.g., supporting the description of complex structures and the transfer of properties to and from structures and their component parts.

Some expressive requirements will, however, call for very expressive ontology languages based on (larger fragments of) FOL, where key reasoning problems are no longer decidable in general, e.g., the recently proposed SWRL language [49]. How to provide practical reasoning support for such languages is still an open problem, but encouraging results have already been obtained using a state-of-the art first order theorem prover with special optimisation and tuning designed to help them cope with the large number of axioms found in realistic ontologies [50].

\subsection{Scalability}

Even for $\mathcal{S H \mathcal { Q }}$, class consistency/subsumption reasoning is ExPTIME-complete, and for $\mathcal{S H O I N}$ this jumps to NExPTIME-complete [26]. There is encouraging evidence of empirical tractability and scalability for implemented DL systems $[34,51]$, but this is mostly w.r.t. logics that do not include inverse properties (e.g., $\left.\mathcal{S} \mathcal{H} \mathcal{F}^{4}\right)$. Adding inverse properties makes practical implementations more problematical as several important optimisation techniques become much less

\footnotetext{
${ }^{4} \mathcal{S H F}$ is equivalent to $\mathcal{S H \mathcal { Q }}$ without inverse properties and with only functional
} properties instead of qualified number restrictions [21]. 
effective. Work is required in order to develop more highly optimised implementations supporting inverse properties, and to demonstrate that they can scale as well as $\mathcal{S H \mathcal { F }}$ implementations. It is also unclear if existing techniques will be able to cope with large numbers of class/property instances [52].

Coping with the large volumes of instance data that will be required by many applications (i.e., millions of individuals) will be extremely challenging, given that existing DL implementations cannot deal with more than (in the order of) a few thousand individuals, even when the relational structure is relatively simple [44]. It seems doubtful that, in the case of instance data, the necessary improvement in performance can be achieved by optimising tableaux based algorithms, which are inherently limited by the need to build and maintain a model of the whole ontology (including all of the instance data).

Several alternative approaches are currently under investigation. One of these involves the use of a hybrid DL-DB architecture in which instance data is stored in a database, and query answering exploits the relatively simple relational structure encountered in typical data sets in order minimise the use of DL reasoning and maximise the use of database operations. A successful prototype of this architecture, the so-called instance store, has already been developed [44]. This prototype is, however, only able to deal with data that has no relational structure (i.e., in which the instance data does not include any role assertions), and so cannot answer queries involving relationships between individuals. Work is underway to extend the prototype to deal with arbitrary instance data, but it is too early to say if this will be successful.

Another technique that is under investigation is to use reasoning techniques based on the encoding of $\mathcal{S H \mathcal { I } Q}$ ontologies in Datalog [53]. On the one hand, theoretical investigations of this technique have revealed that data complexity (i.e., the complexity of answering queries against a fixed ontology and set of instance data) is significantly lower than the complexity of class consistency

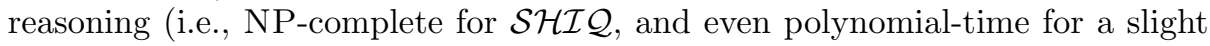
restriction of $\mathcal{S H I Q}$ ) [54]; on the other hand, the technique would allow relatively efficient Datalog engines to be used to store and reason with large volumes of instance data. Again, it is still too early to determine if this technique will be useful in practice.

\subsection{Extended Reasoning Services}

In addition to solving problems of class consistency/subsumption and instance checking, explaining how such inferences are derived may be important, e.g., to help an ontology designer to rectify problems identified by reasoning support, or to explain to a user why an application behaved in an unexpected manner.

Work on developing practical explanation systems is at a relatively early stage, with different approaches still being developed and evaluated. One such technique involves exploiting standard reasoning services to identify a small set of axioms that still support the inference in question, the hope being that presenting a much smaller (than the complete ontology) set of axioms to the user will 
help them to understand the "cause" of the inference [55]. Another (possibly complementary) technique involves explaining the steps by which the inference was derived, e.g., using a sequence of simple natural deduction style inferences $[56,57]$.

As well as explanation, so-called "non-standard inferences" could also be important in supporting ontology design; these include matching, approximation, and difference computations. Non-standard inferences are the subject of ongoing research [58-61]; it is still not clear if they can be extended to deal with logics as expressive as those that underpin modern ontology languages, or if they will scale to large applications ontologies.

\section{Summary}

Description Logics are a family of class based knowledge representation formalisms characterised by the use of various constructors to build complex classes from simpler ones, and by an emphasis on the provision of sound, complete and (empirically) tractable reasoning services. They have been used in a wide range of applications, but perhaps most notably (at least in recent times) in providing a formal basis and reasoning services for (web) ontology languages such as OWL.

The increasing use of DL based ontologies in areas such as e-Science and the Semantic Web is, however, already stretching the capabilities of existing DL systems, and brings with it a range of challenges for future research. The extended ontology languages needed in some applications may demand the use of more expressive DLs, and even for existing languages, providing efficient reasoning services is extremely challenging.

Some applications may even call for ontology languages based on larger fragments of FOL. The development of such languages, and reasoning services to support them, extends these challenges to the whole logic based Knowledge Representation community.

\section{Acknowledgements}

I would like to acknowledge the contribution of the many collaborators with whom I have been privileged to work. These included Franz Baader, Sean Bechhofer, Dieter Fensel, Carole Goble, Frank van Harmelen, Carsten Lutz, Alan Rector, Ulrike Sattler, Peter F. Patel-Schneider, Stephan Tobies and Andrei Voronkov.

\section{References}

1. McGuinness, D.L., Wright, J.R.: An industrial strength description logic-based configuration platform. IEEE Intelligent Systems (1998) 69-77

2. Calvanese, D., De Giacomo, G., Lenzerini, M., Nardi, D., Rosati, R.: Description logic framework for information integration. In: Proc. of the 6th Int. Conf. on Principles of Knowledge Representation and Reasoning (KR'98). (1998) 2-13 
3. Calvanese, D., De Giacomo, G., Lenzerini, M.: On the decidability of query containment under constraints. In: Proc. of the 17th ACM SIGACT SIGMOD SIGART Symp. on Principles of Database Systems (PODS'98). (1998) 149-158

4. Horrocks, I., Tessaris, S., Sattler, U., Tobies, S.: How to decide query containment under constraints using a description logic. In: Proc. of the 7th Int. Workshop on Knowledge Representation meets Databases (KRDB 2000), CEUR (http://ceurws.org/) (2000)

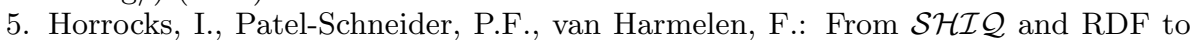
OWL: The making of a web ontology language. J. of Web Semantics 1 (2003) 7-26

6. Knublauch, H., Fergerson, R., Noy, N., Musen, M.: The protégé OWL plugin: An open development environment for semantic web applications. In McIlraith, S.A., Plexousakis, D., van Harmelen, F., eds.: Proc. of the 2004 International Semantic Web Conference (ISWC 2004). Number 3298 in Lecture Notes in Computer Science, Springer (2004) 229-243

7. Liebig, T., Noppens, O.: Ontotrack: Combining browsing and editing with reasoning and explaining for OWL Lite ontologies. In McIlraith, S.A., Plexousakis, D., van Harmelen, F., eds.: Proc. of the 2004 International Semantic Web Conference (ISWC 2004). Number 3298 in Lecture Notes in Computer Science, Springer (2004) 244-258

8. Rector, A.L., Nowlan, W.A., Glowinski, A.: Goals for concept representation in the GALEN project. In: Proc. of the 17th Annual Symposium on Computer Applications in Medical Care (SCAMC'93), Washington DC, USA (1993) 414-418

9. Visser, U., Stuckenschmidt, H., Schuster, G., Vögele, T.: Ontologies for geographic information processing. Computers in Geosciences (to appear)

10. Oberle, D., Sabou, M., Richards, D.: An ontology for semantic middleware: extending daml-s beyond web-services. In: Proceedings of ODBASE 2003. (2003)

11. Wroe, C., Goble, C.A., Roberts, A., Greenwood, M.: A suite of DAML+OIL ontologies to describe bioinformatics web services and data. Int. J. of Cooperative Information Systems (2003) Special Issue on Bioinformatics.

12. Berners-Lee, T., Hendler, J., Lassila, O.: The semantic Web. Scientific American 284 (2001) 34-43

13. The DAML Services Coalition: DAML-S: Web service description for the semantic web. In: Proc. of the 2003 International Semantic Web Conference (ISWC 2003). Number 2870 in Lecture Notes in Computer Science, Springer (2003)

14. Uschold, M., King, M., Moralee, S., Zorgios, Y.: The enterprise ontology. Knowledge Engineering Review 13 (1998)

15. Stevens, R., Goble, C., Horrocks, I., Bechhofer, S.: Building a bioinformatics ontology using OIL. IEEE Transactions on Information Technology in Biomedicine 6 (2002) 135-141

16. Rector, A., Horrocks, I.: Experience building a large, re-usable medical ontology using a description logic with transitivity and concept inclusions. In: Proceedings of the Workshop on Ontological Engineering, AAAI Spring Symposium (AAAI'97), AAAI Press, Menlo Park, California (1997)

17. Spackman, K.: Managing clinical terminology hierarchies using algorithmic calculation of subsumption: Experience with SNOMED-RT. J. of the Amer. Med. Informatics Ass. (2000) Fall Symposium Special Issue.

18. Emmen, A.: The grid needs ontologies-onto-what? http://www.hoise.com/primeur/03/articles/monthly/AE-PR-02-03-7.html.

19. Tuecke, S., Czajkowski, K., Foster, I., Frey, J., Graham, S., Kesselman, C., Vanderbilt, P.: Grid service specification (draft). GWD-I draft, GGF Open Grid Services Infrastructure Working Group (2002) http://www.globalgridforum.org/. 
20. Foster, I., Kesselman, C., Nick, J., Tuecke, S.: The physiology of the grid: An open grid services architecture for distributed systems integration (2002) http://www.globus.org/research/papers/ogsa.pdf.

21. Horrocks, I., Sattler, U., Tobies, S.: Practical reasoning for expressive description logics. In Ganzinger, H., McAllester, D., Voronkov, A., eds.: Proc. of the 6th Int. Conf. on Logic for Programming and Automated Reasoning (LPAR'99). Number 1705 in Lecture Notes in Artificial Intelligence, Springer (1999) 161-180

22. Baader, F., Calvanese, D., McGuinness, D., Nardi, D., Patel-Schneider, P.F., eds.: The Description Logic Handbook: Theory, Implementation and Applications. Cambridge University Press (2003)

23. Blackburn, P., Seligman, J.: Hybrid languages. J. of Logic, Language and Information 4 (1995) 251-272

24. Baader, F., Hanschke, P.: A schema for integrating concrete domains into concept languages. In: Proc. of the 12th Int. Joint Conf. on Artificial Intelligence (IJCAI'91). (1991) 452-457

25. Donini, F.M., Lenzerini, M., Nardi, D., Nutt, W.: The complexity of concept languages. Information and Computation 134 (1997) 1-58

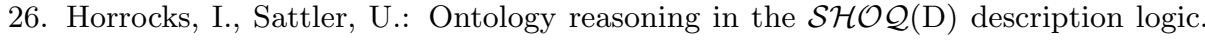
In: Proc. of the 17th Int. Joint Conf. on Artificial Intelligence (IJCAI 2001). (2001) 199-204

27. Horrocks, I.: The FaCT system. In de Swart, H., ed.: Proc. of the 2nd Int. Conf. on Analytic Tableaux and Related Methods (TABLEAUX'98). Volume 1397 of Lecture Notes in Artificial Intelligence., Springer (1998) 307-312

28. Patel-Schneider, P.F.: DLP system description. In: Proc. of the 1998 Description Logic Workshop (DL'98), CEUR Electronic Workshop Proceedings, http://ceurws.org/Vol-11/ (1998) 87-89

29. Haarslev, V., Möller, R.: RACER system description. In: Proc. of the Int. Joint Conf. on Automated Reasoning (IJCAR 2001). Volume 2083 of Lecture Notes in Artificial Intelligence., Springer (2001) 701-705

30. Pan, J.Z.: Description Logics: Reasoning Support for the Semantic Web. PhD thesis, University of Manchester (2004)

31. Horrocks, I., Sattler, U., Tobies, S.: Practical reasoning for very expressive description logics. J. of the Interest Group in Pure and Applied Logic 8 (2000) 239-264

32. Baader, F., Franconi, E., Hollunder, B., Nebel, B., Profitlich, H.J.: An empirical analysis of optimization techniques for terminological representation systems or: Making KRIS get a move on. Applied Artificial Intelligence. Special Issue on Knowledge Base Management 4 (1994) 109-132

33. Bresciani, P., Franconi, E., Tessaris, S.: Implementing and testing expressive description logics: Preliminary report. In: Proc. of the 1995 Description Logic Workshop (DL'95). (1995) 131-139

34. Horrocks, I.: Using an expressive description logic: FaCT or fiction? In: Proc. of the 6th Int. Conf. on Principles of Knowledge Representation and Reasoning (KR'98). (1998) 636-647

35. Patel-Schneider, P.F.: DLP. In: Proc. of the 1999 Description Logic Workshop (DL'99), CEUR Electronic Workshop Proceedings, http://ceur-ws.org/Vol22/ (1999) 9-13

36. Horrocks, I., Patel-Schneider, P.F.: Optimizing description logic subsumption. J. of Logic and Computation 9 (1999) 267-293 
37. Horrocks, I., Tobies, S.: Reasoning with axioms: Theory and practice. In: Proc. of the 7th Int. Conf. on Principles of Knowledge Representation and Reasoning (KR 2000). (2000) 285-296

38. Baker, A.B.: Intelligent Backtracking on Constraint Satisfaction Problems: Experimental and Theoretical Results. PhD thesis, University of Oregon (1995)

39. Oppacher, F., Suen, E.: HARP: A tableau-based theorem prover. J. of Automated Reasoning 4 (1988) 69-100

40. Wroe, C., Stevens, R., Goble, C.A., Ashburner, M.: A methodology to migrate the Gene Ontology to a description logic environment using DAML+OIL. In: Proc. of the 8th Pacific Symposium on Biocomputing (PSB). (2003)

41. Rogers, J.E., Roberts, A., Solomon, W.D., van der Haring, E., Wroe, C.J., Zanstra, P.E., Rector, A.L.: GALEN ten years on: Tasks and supporting tools. In: Proc. of MEDINFO2001. (2001) 256-260

42. Rector, A.: Analysis of propagation along transitive roles: Formalisation of the galen experience with medical ontologies. In: Proc. of DL 2002, CEUR (http://ceur-ws.org/) (2002)

43. Schulz, S., Hahn, U.: Parts, locations, and holes - formal reasoning about anatomical structures. In: Proc. of AIME 2001. Volume 2101 of Lecture Notes in Artificial Intelligence., Springer (2001)

44. Horrocks, I., Li, L., Turi, D., Bechhofer, S.: The instance store: DL reasoning with large numbers of individuals. In: Proc. of the 2004 Description Logic Workshop (DL 2004). (2004) 31-40

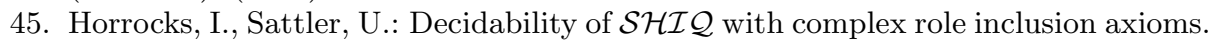
Artificial Intelligence 160 (2004) 79-104

46. Lutz, C., Areces, C., Horrocks, I., Sattler, U.: Keys, nominals, and concrete domains. J. of Artificial Intelligence Research (2004) To Appear.

47. Wolter, F., Zakharyaschev, M.: Temporalizing description logics. In Gabbay, D., de Rijke, M., eds.: Frontiers of Combining Systems II. Studies Press/Wiley (2000) 379-401

48. Pan, J.Z., Horrocks, I.: Extending Datatype Support in Web Ontology Reasoning. In: Proc. of the 2002 Int. Conference on Ontologies, Databases and Applications of SEmantics (ODBASE 2002). Number 2519 in Lecture Notes in Computer Science, Springer (2002) 1067-1081

49. Horrocks, I., Patel-Schneider, P.F., Boley, H., Tabet, S., Grosof, B., Dean, M.: SWRL: A semantic web rule language combining owl and ruleml. W3C Member Submission (2004) Available at http://www.w3.org/Submission/SWRL/.

50. Tsarkov, D., Riazanov, A., Bechhofer, S., Horrocks, I.: Using Vampire to reason with OWL. In McIlraith, S.A., Plexousakis, D., van Harmelen, F., eds.: Proc. of the 2004 International Semantic Web Conference (ISWC 2004). Number 3298 in Lecture Notes in Computer Science, Springer (2004) 471-485

51. Haarslev, V., Möller, R.: High performance reasoning with very large knowledge bases: A practical case study. In: Proc. of the 17th Int. Joint Conf. on Artificial Intelligence (IJCAI 2001). (2001) 161-168

52. Horrocks, I., Sattler, U., Tobies, S.: Reasoning with individuals for the description

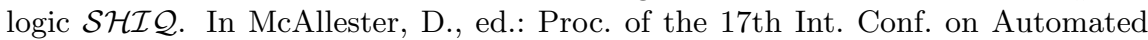
Deduction (CADE 2000). Volume 1831 of Lecture Notes in Computer Science., Springer (2000) 482-496

53. Hustadt, U., Motik, B., Sattler, U.: Reducing SHIQ-description logic to disjunctive datalog programs. In: Proc. of the 9th Int. Conf. on Principles of Knowledge Representation and Reasoning (KR 2004). (2004) 152-162 
54. Motik, B., Sattler, U., Studer, R.: Query answering for OWL-DL with rules. In: Proc. of the 2004 International Semantic Web Conference (ISWC 2004). (2004) 549-563

55. Schlobach, S., Cornet, R.: Explanation of terminological reason-ing: A preliminary report. In: Proc. of the 2003 Description Logic Workshop (DL 2003). (2003)

56. McGuinness, D.L.: Explaining Reasoning in Description Logics. PhD thesis, Rutgers, The State University of New Jersey (1996)

57. Borgida, A., Franconi, E., Horrocks, I.: Explaining $\mathcal{A L C}$ subsumption. In: Proc. of the 14th Eur. Conf. on Artificial Intelligence (ECAI 2000). (2000)

58. Baader, F., Küsters, R., Borgida, A., McGuinness, D.L.: Matching in description logics. J. of Logic and Computation 9 (1999) 411-447

59. Brandt, S., Turhan, A.Y.: Using non-standard inferences in description logics what does it buy me? In: Proc. of KI-2001 Workshop on Applications of Description Logics (KIDLWS'01). Volume 44 of CEUR (http://ceur-ws.org/). (2001)

60. Küsters, R.: Non-Standard Inferences in Description Logics. Volume 2100 of Lecture Notes in Artificial Intelligence. Springer Verlag (2001)

61. Brandt, S., Küsters, R., Turhan, A.Y.: Approximation and difference in description logics. In: Proc. of the 8th Int. Conf. on Principles of Knowledge Representation and Reasoning (KR 2002). (2002) 203-214 